\title{
Different approaches for multi-band transport in semiconductors
}

\author{
Giovanni Borgioli and Omar Morandi
}

Dipartimento di Elettronica e Telecomunicazioni

Università degli Studi di Firenze, I-50139 Firenze, Italy

Giovanni Frosali

Dipartimento di Matematica "G. Sansone"

Università degli Studi di Firenze, I-50139 Firenze, Italy

Michele Modugno

Dipartimento di Fisica

Università degli Studi di Firenze, I-50019 Firenze, Italy

Most recent nanometric semiconductor devices like RITDS (Resonant interband tunneling diodes) [1,2] exhibit a full quantum behaviour which can be exhaustively understood only starting from the Schrödinger equation. Yet, this "exact", formulation is too complicated to deal with, since the Hamiltonian should take in account the external field potential and the potential barriers due to the heterogeneous structure of the device besides the periodic potential of the crystal (we disregard in our models the field due to impurities, magnetic fields and electronic spin).

In this paper we are interested to perform and compare two simplified models, deduced from the Schrödinger equation, which are able to simulate interband tunneling between conduction and the upper (not degenerate) valence band. The tool is an expansion of the full wave function in a suitable orthonormal and complete $L^{2}$ basis. The "coefficients" of this expansion are the so-called envelope functions, which represent a smoothed version of the wave function. The Kane model [3] is obtained choosing as a basis the periodic part of the Bloch functions, the so-called Wannier basis, $u_{n, k}(x)$ [4] evaluated at the point $k=0$, where it is assumed that the minimum for the conduction band and the maximum for the valence band in the crystal momentum representation occur ( $n$ is the band index and in the case here faced we have $n=c, v)$. The Kane model is represented by a system of two Schrödinger-like equations (here we limit ourselves to the one-dimensional formulation):

$$
\left\{\begin{aligned}
i \hbar \frac{\partial \Psi_{c}^{\text {Kane }}}{\partial t} & =-\frac{\hbar^{2}}{2 m} \frac{\partial^{2} \Psi_{c}^{\text {Kane }}}{\partial x^{2}}+\mathcal{E}_{c} \Psi_{c}^{\text {Kane }}-\frac{\hbar^{2}}{m} P \frac{\partial \Psi_{v}^{\text {Kane }}}{\partial x} \\
i \hbar \frac{\partial \Psi_{v}^{\text {Kane }}}{\partial t} & =-\frac{\hbar^{2}}{2 m} \frac{\partial^{2} \Psi_{v}^{\text {Kane }}}{\partial x^{2}}+\mathcal{E}_{v} \Psi_{v}^{\text {Kane }}+\frac{\hbar^{2}}{m} P \frac{\partial \Psi_{c}^{\text {Kane }}}{\partial x}
\end{aligned}\right.
$$

where $\Psi_{c}^{\text {Kane }}$ and $\Psi_{v}^{\text {Kane }}$ are, respectively, the conduction and valence band envelope functions (in the Kane representation), $i$ is the imaginary unit, $\hbar$ is the reduced Planck constant, $m$ is the bare mass of the carriers, $\mathcal{E}_{c}$ and $\mathcal{E}_{v}$ are the minimum of 
the conduction band energy and maximum of the valence band energy respectively, including the effect of the external field, and $P$ is the coupling coefficient between the two bands. The Kane model has been discussed in a recent paper [5] and its Wigner formulation has been performed in [6].

In the second part of the paper we use a similar, but different approach in order to perform a possibly alternative model. We start choosing another basis where to project the wave function, which can be considered a better approximation of Wannier basis than the one supplied by Kane basis. We denote this basis as Kohn basis $[7,8]$ and its elements are given by:

$$
u_{n, k}^{K o h n}=u_{n, 0}+\left.\frac{\partial u_{n, k}}{\partial k}\right|_{k=0} k .
$$

The system of Schrödinger-like differential equations obtained for Kohn envelope functions writes as follows

$$
\left\{\begin{aligned}
i \hbar \frac{\partial \Psi_{c}^{K o h n}}{\partial t} & =-\frac{\hbar^{2}}{2 m^{*}} \frac{\partial^{2} \Psi_{c}^{K o h n}}{\partial x^{2}}+E_{c} \Psi_{c}^{K o h n}-\frac{\hbar^{2}}{m} \frac{P}{E_{g}} \frac{\partial V}{\partial x} \Psi^{K o h n} \\
i \hbar \frac{\partial \Psi_{v}^{K o h n}}{\partial t} & =\frac{\hbar^{2}}{2 m^{*}} \frac{\partial^{2} \Psi_{v}^{K o h n}}{\partial x^{2}}+E_{v} \Psi_{v}^{K o h n}-\frac{\hbar^{2}}{m} \frac{P}{E_{g}} \frac{\partial V}{\partial x} \Psi_{c}^{K o h n}
\end{aligned}\right.
$$

where $\Psi_{c}^{K o h n}$ and $\Psi_{v}^{K o h n}$ are, respectively, the conduction and valence band envelope functions (in the Kohn representation), $E_{c}$ and $E_{v}$ are the unperturbed minimum of the conduction band energy and maximum of the valence band energy respectively, $m^{*}$ is the effective mass of the carriers, $E_{g}=E_{c}-E_{v}$ and $V$ is the external potential.

The final perspective of the work is to develop numerical simulations for both models and compare them in order to test their possible range of validity.

\section{References}

[1] M. Sweeney and J. M. Xu, Resonant interband tunnel diodes, Appl. Phys. Lett. 54 (6), 546-548 (1989).

[2] R.Q. Yang, M. Sweeny, D. Day and J.M. Xu, Interband tunneling in heterostructure tunnel diodes, IEEE Transactions on Electron Devices, 38(3) 442446 (1991).

[3] E. O. Kane, Energy band structure in p-type Germanium and Silicon, J. Phys. Chem. Solids 1, 82-89 (1956).

[4] W.T. Wenckebach, Essential of Semiconductor Physics, J.Wiley \& Sons, Chichester, 1999. 
[5] S. Biondini and G. Borgioli, Rigorous derivation of Kane models for interband tunneling, Proceedings of WASCOM 2003-XII Conference on Waves and Stability in Continuous Media, Eds. R. Monaco, S: Pennisi, S. Rionero and T. Ruggeri, World Scientific, Singapore, 70-77 (2004).

[6] G. Borgioli, G. Frosali, P.F. Zweifel, Wigner approach to the two-band Kane model for a tunneling diode, Transport Theory Stat. Phys., 32, 3 \& 4, 347-366 (2003).

[7] E.N. Adams II, Motion of an electron in a perturbed periodic potential, Physical Review, 85, 1, 41-50 (1952).

[8] J.M. Luttinger and W. Kohn, Motion of electrons and holes in perturbed periodic fields, Physical Review, 9, 4, 869-883 (1954). 